\title{
Standard Risk Acute Leukemia
}

National Cancer Institute

\section{Source}

National Cancer Institute. Standard Risk Acute Leukemia. NCI Thesaurus. Code C122457.

Acute leukemia patients are stratified into the standard risk group when their minimal residual disease levels are lower than $0.05 \%$. 